\title{
Centerline Correction for Precision Roughness Specimens
}

\section{J. L. Chamberlin}

\author{
(May 24, 1965)
}

\begin{abstract}
In graphic determinations of roughness height (a measure of surface texture) a specific centerline about which the total area is measured is required. The first approximation of the position of this centerline is subjective and a repositioning was generally required. Here presented is a method for determining the roughness height without resorting to an additional area measurement.
\end{abstract}

Key Words: Centerline corrections, precision roughness specimens, roughness height.

Roughness height, one measure of the texture of a surface, is the arithmetic average deviation of the undulations of the surface, and is expressed by

$$
R_{a}=\frac{1}{L} \int_{0}^{L}|y| d x
$$

where

$R_{a}$ is the roughness height,

$L$ is the length over which the roughness height is measured,

$y$ is the amplitude of the undulations measured normal to their centerline, and

$d x$ is the infinitesimal length measured along the centerline.

For uniform repetitive wave forms. like those comprising a precision roughness specimen, the centerline is taken as a line parallel to the general direction of the profile section of the surface tested, and so positioned that the area contained between it and those portions of the surface's profile which lie above it are equal to the area between it and the profile portions lying below it.

The length over which the roughness height is determined is generally taken as equal to a roughnesswidth cutoff or some whole multiple thereof. The roughness-width cutoff is the greatest spacing of repetitive surface irregularities which are to be included in the measurement for roughness height.

In the calibration of precision roughness specimens a graphical interpretation of roughness height is often employed. This results from performing the indicated integration of the roughness height defining eq (1). This integrated form is

$$
R_{a}=\frac{1}{L}\left(A_{T}+A_{B}\right)
$$

where

$A_{T}$ is the area between the centerline and that portion of the profile lying above it;

$A_{B}$ is the area between the centerline and that portion of the profile lying below it.

In practice the surface to be measured is recorded using a surface finish analyzer. Such analyzers slowly traverse a sharp stylus across the surface. The undulation of this stylus relative to a straight datum is amplified and recorded, resulting in a magnified profile section of the surface. A centerline is positioned on this recording by visual estimation, and the length over which roughness height is to be determined is marked off to the nearest whole cycle of the undulations. The areas above and below the centerline are then planimetrically determined. If the measured areas are the same then application of eq (2), being mindful of the appropriate recorder magnifications, yields the roughness height. If the measured areas are not the same then it has been general practice to reposition the centerline and redetermine the areas.

If three conditions are met, this remeasurement may be circumvented by making a correction for the malplacement of the centerline. These conditions are as follows: First, the profile traced is comprised of uniform, repetitive wave forms; second, a minimal amount of waviness is present; and third, the difference between measured areas is small compared to their sum. Waviness is the long wavelength undulations of the surface upon which the repetitive wave forms are superimposed.

For consideration as a precision roughness specimen the first and second conditions must exist. The third condition follows as a natural consequence of the first two conditions since the more uniform the specimen, the easier it is to position the centerline. On typical specimens it is common to have the difference in areas less than 5 percent of the total area on the first visual estimation of the centerline position. 
The required correction also is obtained as a consequence of the above conditions. That is, the roughness height of a specimen may be obtained from the measured areas and a knowledge of other surface parameters. In this development the measured values are primed and the desired values are not. Thus, the total measured area, $A^{\prime}$, is

$$
A^{\prime}=A_{T}^{\prime}+A_{B}^{\prime}
$$

and the desired total area for the roughness height, $A$, is

$$
A=A_{T}+A_{B} .
$$

The error, $E$, in determining the correct area for application of eq (2) is

$$
E=A-A^{\prime}=A_{T}+A_{B}-\left(A_{T}^{\prime}+A_{B}^{\prime}\right) .
$$

The total correction is equal to the sum of the individual corrections of each cycle making up the length tested, or $n$ times the average correction where $n$ is the number of cycles in the length investigated. In this development figure $l$ is to represent the average cycle and the corrections are developed for this case.

For the small correction to the total area involved it is sufficient to consider areas $a^{\prime} a b b^{\prime}$ and $b^{\prime} b c c^{\prime}$ as trapezoids with altitude $\Delta y$.

The area above the centerline is for a single cycle

$$
a_{T}=a_{T}^{\prime}-\frac{\Delta y}{2}\left[\left(b^{\prime}-a^{\prime}\right)+(b-a)\right]
$$

and below the centerline

$$
a_{B}=a_{B}^{\prime}+\frac{\Delta y}{2}\left[\left(c^{\prime}-b^{\prime}\right)+(c-b)\right]
$$

where $\Delta y$ is positive when

$$
A_{T}^{\prime}>A_{B}^{\prime}
$$

(The lower case represents a measure referred to the single average cycle.) The error is thus

$$
\begin{aligned}
E=n\left\{-\frac{\Delta y}{2}\left[\left(b^{\prime}-a^{\prime}\right)+(b-a)\right]\right. & \\
& \left.+\frac{\Delta y}{2}\left[\left(c^{\prime}-b^{\prime}\right)+(c-b)\right]\right\} .
\end{aligned}
$$

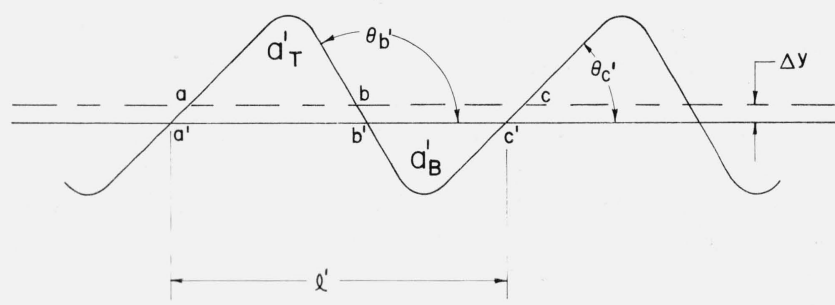

FIGURE 1. Representation of the average cycle of a precision roughness specimen as measured about a malplaced centerline.
But

$$
\begin{aligned}
& (b-a)=(c-a)-(c-b) \\
& \left(b^{\prime}-a^{\prime}\right)=\left(c^{\prime}-a^{\prime}\right)-\left(c^{\prime}-b^{\prime}\right) \\
& \left(c^{\prime}-a^{\prime}\right)=(c-a)=l^{\prime}=L^{\prime} / n
\end{aligned}
$$

and

$$
(c-b)=\left(c^{\prime}-b^{\prime}\right)+\Delta y\left(\cot \theta_{c^{\prime}}-\cot \theta_{b^{\prime}}\right) .
$$

Thus, the error becomes, upon substitution of eqs (9),

$$
E=n\left\{-\Delta y\left[l^{\prime}-2\left(c^{\prime}-b^{\prime}\right)-\Delta y\left(\cot \theta_{c^{\prime}}-\cot \theta_{b^{\prime}}\right)\right]\right\}(10)
$$

By definition

$$
A_{T}-A_{B}=0
$$

and

$$
A_{T}^{\prime}-A_{B}^{\prime}=\Delta A^{\prime} .
$$

Therefore the measured area difference is

$$
\Delta A^{\prime}=\frac{n \Delta y}{2}\left[\left(b^{\prime}-a^{\prime}\right)+(b-a)+\left(c^{\prime}-b^{\prime}\right)+(c-b)\right]
$$

or upon reduction

$$
\Delta A^{\prime}=L^{\prime} \Delta y .
$$

The area required for the roughness determination is therefore

$A=A^{\prime}-\frac{\Delta A^{\prime}}{L^{\prime}} n\left[l^{\prime}-2\left(c^{\prime}-b^{\prime}\right)\right.$

$$
\left.-\frac{\Delta A^{\prime}}{L^{\prime}}\left(\cot \theta_{c^{\prime}}-\cot \theta_{b^{\prime}}\right)\right]
$$

The desired roughness height based on measurements made with a malplaced centerline is therefore

$R_{a}=R_{a^{\prime}}-\frac{\Delta A^{\prime}}{L^{\prime}}$

$$
\left[1-\frac{2\left(c^{\prime}-b^{\prime}\right)}{L^{\prime} / n}-\frac{\Delta A^{\prime}}{L^{\prime}} \frac{\left(\cot \theta_{c^{\prime}}-\cot \theta_{b^{\prime}}\right)}{L^{\prime} / n}\right]
$$

In practice the parameters of the average cycle used in the adjustment of the roughness height are the averages of individual values measured at points randomly chosen along the trace. Because of the uniformity of most precision roughness specimens it is satisfactory to use the cotangent of the average angle rather than the average cotangent of the angles. For example, the cotangent of the average angle and the average cotangent of the angles for four precision roughness specimens that were being tested during 
the writing of this paper are shown in table 1 . The values given are for five individual measurements made along the recorded trace. The recorded traces contained eight cycles for specimens 1 and 2 and ten cycles for specimens 3 and 4 .

TABLE 1

\begin{tabular}{c|c|c|c|c|c|c}
\hline \hline & & & & & \\
Specimen & $\cot \bar{\theta}_{b^{\prime}}$ & $\overline{\cot } \theta_{b^{\prime}}$ & Range of $\theta_{b^{\prime}}$ & $\cot \bar{\theta}_{c^{\prime}}$ & $\overline{\cot } \theta_{c^{\prime}}$ & Range of $\theta_{c^{\prime}}$ \\
\hline & & & Degree & & & Degree \\
1 & -0.895 & -0.896 & 3.2 & 1.155 & 1.155 & 1.5 \\
2 & -.887 & -.888 & 2.4 & 1.108 & 1.111 & 5.9 \\
3 & -.202 & -.202 & 2.2 & 0.233 & 0.233 & 1.5 \\
4 & -.186 & -.186 & 2.2 & .246 & .247 & 4.1 \\
\hline
\end{tabular}

To illustrate the use of the correcting equation, the roughness height of a trace similar to that shown in figure 2 was measured using a visually estimated centerline and four malplaced lines. The malplaced lines were approximately 0.05 and $0.10 \mathrm{in}$. above and below the centerline. The results are tabulated in table 2.

TABLE 2

\begin{tabular}{|c|c|c|c|c|c|c|c|c|}
\hline Position & $A_{T}$ & $A_{k^{\prime}}$ & $L^{\prime}$ & $\left(\overline{c^{\prime}-b^{\prime}}\right)$ & $\cot \bar{\theta}_{b}$ & $\cot \bar{\theta}_{r^{\prime}}$ & $R_{a^{\prime}}$ & $R_{a}$ \\
\hline & sq. inches & sq. inches & Inches & Inch & & & Inch & Inch \\
\hline 0.10 in. above & 1.378 & 2.123 & 7.523 & 0.512 & -0.1475 & 0.3344 & 0.465 & $0.46 l_{6}$ \\
\hline 0.05 in. above & 1.612 & 1.872 & 7.521 & .483 & -.1514 & .3211 & $.463_{2}$ & $.462 y$ \\
\hline Centerline & 1.775 & 1.704 & 7.524 & .462 & -.1530 & .3213 & $.462_{4}$ & $.462_{4}$ \\
\hline 0.05 in. below & 1.932 & 1.567 & 7.527 & .442 & -.1534 & .3276 & $.464 y$ & $.463_{1}$ \\
\hline 0.10 in. below & 2.131 & 1.383 & 7.526 & .421 & -.1562 & .3325 & $.466_{9}$ & $.461_{6}$ \\
\hline
\end{tabular}

Each of the average values is based on eight individual measurements. The trace contained eight cycles.

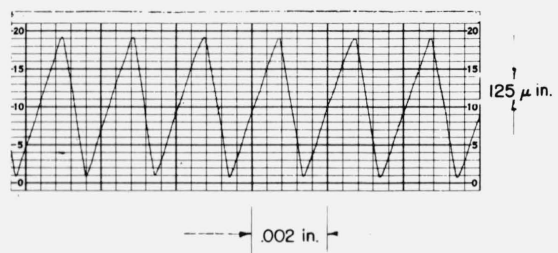

FIGURE 2. Typical trace of the precision roughness specimen used in illustrating the correcting equation.

To obtain the specimen's roughness height, the roughness height obtained from measurement on the recorded trace needs to be divided by the vertical magnification of the recorder. In this example, the vertical magnification was $2112 \times$.

In this example $24 \mathrm{~min}$ were required to position a line and measure the areas about it. It required 17 min to measure the various parameters and to calculate the correction. A time savings of 29 percent could have been achieved in this case over repositioning for a malplaced centerline.

The use of this correcting equation has eliminated the centerline repositioning for graphic roughness height determinations. The time required to measure the necessary parameters is less than that required to reposition the centerline and make a new planimetric determination. Depending upon the complexity of the trace being measured, time savings up to 75 percent are possible.

(Paper 69C4-211) 